\title{
Meningeal Carcinomatosis
}

National Cancer Institute

\section{Source}

National Cancer Institute. Meningeal Carcinomatosis. NCI Thesaurus. Code C27383.

Diffuse spread of cancer to the meninges. 